\title{
Parasympathetic Characterization Guided by Respiration From Wrist Peripheral Venous Pressure Waveform
}

\author{
Diego Cajal ${ }^{1,2}$, David Hernando ${ }^{1,2}$, Jesús Lázaro ${ }^{1,2}$, Eduardo Gil ${ }^{1,2}$, Annie Alvis ${ }^{3}$, Monica Polcz ${ }^{3,4}$, \\ Bret Alvis ${ }^{4}$, Kyle Hocking ${ }^{3}$, Colleen Brophy ${ }^{3}$, Raquel Bailón ${ }^{1,2}$ \\ ${ }^{1}$ BSICoS Group, Aragón Institute of Engineering Research (I3A), IIS Aragón, University of \\ Zaragoza, Zaragoza, Spain \\ ${ }^{2}$ CIBER - Bioingeniería, Biomateriales y Nanomedicina (CIBER-BBN), Madrid, Spain \\ ${ }^{3}$ VoluMetrix, Nashville TN, USA \\ ${ }^{4}$ Vanderbilt University Medical Center, Nashville TN, USA
}

\begin{abstract}
VoluMetrix has developed a new version of its NIVA, a wrist device that measures pressure variations in the veins together with the photoplethysmogram (PPG) at the same point. Previous studies have shown that the venous pressure signal (NIVA) reflected an increased HF power with respect to the electrocardiogram. This suggests that it may be useful for parasympathetic characterization guided by signal-derived respiration. Performance of NIVA signal is compared to that of PPG in a controlled breathing experiment ( 8 subjects) with different respiratory rates $(6,12$ and $18 \mathrm{bpm})$, where a downward trend in parasympathetic estimates is expected with increasing respiratory rates. The NIVA signal is able to accurately estimate the respiratory rate (less than $0.03 \mathrm{~Hz}$ estimation error) in all the subjects, outperforming the PPG in the same task. In addition, respiratory-guided parasympathetic estimates significantly decreases with increased respiratory rate.
\end{abstract}

\section{Introduction}

Heart Rate Variability (HRV) has been widely studied for several decades as a non-invasive method for autonomic nervous system assessment. HRV is derived from electrocardiographic (ECG) signals, although other signals have been gradually included as surrogates for this purpose, especially with the proliferation of wearable devices. Probably, the most common used signal in these devices is the pulse photoplethysmographic (PPG) signal, which can be used for measuring pulse rate variability (PRV). PRV is not an exact surrogate of HRV but includes information related to the vascular system (e.g., pulse transit time and pulse morphology). However, PRV and HRV are wellknown to be highly correlated [1,2].

Another technology used in some wearable devices is the piezoelectrography, which measures the pressure variations on the surface of the skin. VoluMetrix is performing a Non-Invasive Venous waveform Analysis (NIVA), by developing a device with piezoelectric sensors placed in the volar aspect of the wrist. The development of this device was motivated by some recent results of peripheral intra venous analysis that showed it is a promising indicator in blood volume assessment and hemorrhages [3,4]. The research of Hernando et al $[5,6]$ has demonstrated that NIVA is a reliable surrogate of HRV under resting conditions. In addition, they found a significantly higher power within the High Frequency (HF) band at NIVA, defined as the band centered at the respiratory rate (RR). It suggests that NIVA may provide enhanced respiration information, maybe because venous return is strongly mediated by negative pressure in the chest caused by inspiration movements. VoluMetrix has improved its device by using a new sensor that measures pressure changes (i.e., a pressure derivative) in the vein for NIVA, and by adding a PPG sensor. Classical HRV analysis can be more effective by including respiratory information, as it has been reported for some applications including stress assessment [7]. Thus, the NIVA device could make use of its novel signal to obtain enhanced information on parasympathetic tone guided by respiration, which could be used in stress and wellbeing identification applications (e.g., yoga, mindfulness, etc.) using a wrist device, the most accepted on the market nowadays.

The aim of this work is to continue with the study of the NIVA signal, analyzing its ability to obtain respirationguided parasympathetic markers. First, respiratory information and pulse rate was derived from the signal, and then used to compute the power of PRV in a bandwidth dependent on respiration. Same measurements were obtained from the PPG recorded by the NIVAband as a comparison. Performance will be evaluated in a breathing-controlled 
experiment with different respiratory rates. It is known that, for the same subject, the power in the band defined by respiration tends to decrease with an increase in the RR [8]. Our hypothesis is that the NIVA signal could be more sensitive than the PPG signal to these intra-subject variations.

\section{Materials and methods}

\subsection{Database}

In collaboration with Vanderbilt University Medical Center and under a COI IRB approval, eight healthy volunteers were recorded with the NIVA device, acquiring both NIVA and PPG signals, while maintaining controlled respiration at $0.1,0.2$ and $0.3 \mathrm{~Hz}(6,12$ and $18 \mathrm{bpm}$, respectively). Each stage lasted for 5 to 6 minutes and the subjects were seated throughout the experiment. The NIVA signal is acquired using a piezoelectric sensor that samples at $500 \mathrm{~Hz}$, while the PPG using an optoelectric one at 125 $\mathrm{Hz}$, both in the volar aspect of the wrist.

\subsection{Preprocessing}

All the signals were low pass filtered with a cut-off frequency of $5 \mathrm{~Hz}$ at NIVA and $2 \mathrm{~Hz}$ in the case of PPG (empirically selected according to the different signal to noise ratios) in order to remove high frequency noise; and high pass filtered with a cut-off frequency of $0.5 \mathrm{~Hz}$ to remove low frequency oscillations such as baseline wander. The results have also been calculated for NIVA filtered at $2 \mathrm{~Hz}$ for better comparison.

\subsection{Fiducial points}

We describe now the fiducial points selected for the next steps. These points should be the same temporal points in both signals to obtain a fair comparison. We have chosen the signal onset $\left(t_{O}(k)\right.$, for $\mathrm{k}=1,2, \ldots, \mathrm{K}$, being $\mathrm{K}$ the total number of pulses), and the maximum upslope $\left(t_{U}(k)\right)$ as defined in [9]; and the derivative zero-crossing $\left(t_{A}(k)\right)$. This last point is used instead of the signal endset, described in the same article, because it is a more stable point due to the morphology of the signals. It is important to notice, as depicted in Figure 1, that in the NIVA case, the maximum upslope and derivative zero-crossing are the maximum and zero-crossing of the signal itself.

\subsection{RR estimation}

Two derived respiration techniques were used to calculate the RR: PRV and Slope Transit Time (STT). PRV is computed from $t_{U}(k)$, using the IPFM model [10] and a sampling rate of $4 \mathrm{~Hz}$. STT is computed as
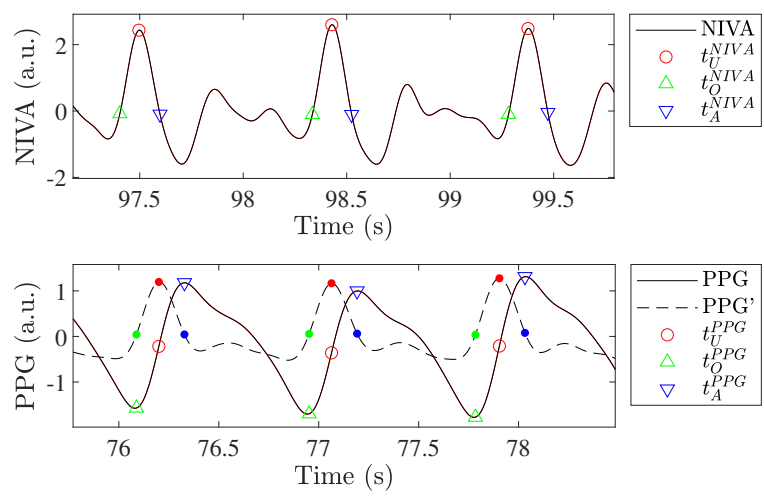

Figure 1: Above: NIVA signal with fiducial points. Below: PPG signal and its derivative (dashed line, augmented) with fiducial points.

$$
S T T=\sum_{k=1}^{K}\left(t_{A}(k)-t_{O}(k)\right) \delta\left(t-t_{A}(k)\right)
$$

An outlier detector based on [11] is used before spline interpolation at $4 \mathrm{~Hz}$.

We chose the respiratory peak as the maximum in a window between 0.05 and $0.4 \mathrm{~Hz}$ in the Power Spectral Density (PSD) of both PRV and STT. The spectral estimation is done by the Welch method, using a 60 second Hamming window, and $50 \%$ overlap. Since no respiratory signal is available, we consider as a success if the estimate deviates less than $0.03 \mathrm{~Hz}$ above or below the metronome frequency. Thus, we name success rate as the ratio of correct estimates to total cases.

\subsection{Respiration-guided parasympathetic as- sessment}

For HF bounds calculation, we have used three different methods. As it will be shown in the results section, the RR estimate obtained from the PRV is chosen for the three methods as it is more robust than the STT one. The first one consists of $0.1 \mathrm{~Hz}$ width windows centered on the estimated RR. The second method centers the band in the same way, but adjusts the bandwidth to $-3 \mathrm{~dB}$ from the peak, instead of a fixed width. The last one is based on spectral coherence between PRV and STT [12]. Spectral coherence is a function of frequency with values between 0 and 1 . It measures the linear relationship between two signals at each specific frequency. As STT is strongly modulated by respiratory rhythms, we obtain the frequencies in which breathing is affecting the heart rate (actually the pulse rate), looking for a high coherence. HF window bounds are evaluated by looking for the frequencies whose coherence is above a threshold around the estimated RR 

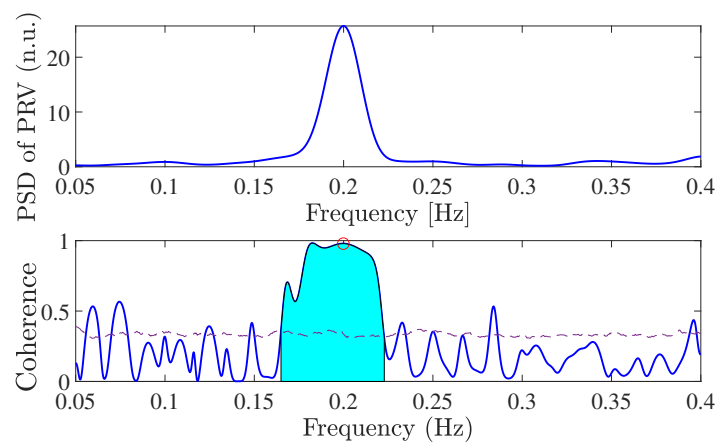

Figure 2: Above: PSD of PRV. The peak around $0.2 \mathrm{~Hz}$ is the one corresponding to respiration. Below: Coherence between PRV and STT. HF window bounds are found by looking for the points that cross the threshold (dashed line) on each side of the peak. The shaded area indicates the window found in this example. This window is used in the upper signal to compute $P_{H F}$.

(see Figure 2). This threshold was previously computed as the 95th percentile of a hundred white gaussian noise realizations. This way, we assure that the coherence is statistically significant. Windows less than $0.015 \mathrm{~Hz}$ wide are rejected and HF power $\left(P_{H F}\right)$ is not assessed. Once the window boundaries are obtained, $P_{H F}$ is calculated in all the cases from the PRV spectrum, using trapezoidal integration. We have normalized the spectrum units by dividing by the power integral between 0.03 and $0.4 \mathrm{~Hz}$.

\subsection{Statistical analysis}

A Wilcoxon signed-rank test is performed, where we test the hypothesis of zero median for the difference between paired samples from different RRs. Thus, we can compare the parasympathetic estimates, obtaining a quantitative measurement of the discrimination between different breathing rates using both signals. A significance level of 0.05 is used to reject or accept the null hypothesis

\section{Results}

We start by analyzing the results of the RR estimation. Using the spectrum of the PRV, we obtain a $100 \%$ success rate for the case of NIVA, filtered at both 5 and $2 \mathrm{~Hz}$. The Root-Mean-Square Error (RMSE) of the estimate from the metronome rate is 0.7 and $1.1 \mathrm{mHz}$ at 5 and $2 \mathrm{~Hz}$, respectively. For PPG, the success rate drops to $87.5 \%$, although the error remains low in the correct cases: $1.3 \mathrm{mHz}$. On the other hand, using the STT spectrum the results are not so good. For both filtered NIVA at 5 and $2 \mathrm{~Hz}$, a success rate of $66.7 \%$ is obtained, with a RMSE of 6 and $6.8 \mathrm{mHz}$. In PPG, the success rate is $75 \%$ and the RMSE is $1 \mathrm{mHz}$.
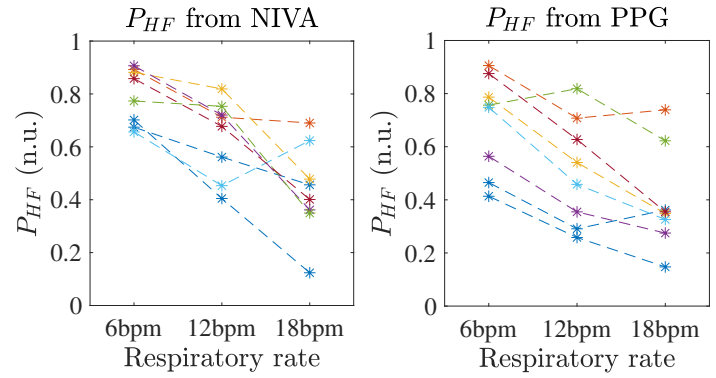

Figure 3: $P_{H F}$ trends with the variation of RR. On the left the trends obtained with the NIVA signal and on the right with PPG. Although downward trends are observed in both, it can be seen that in NIVA the three groups of 6 , 12 and $18 \mathrm{bpm}$ are better separated.

We now turn to analyzing trends in $P_{H F}$. Using fixedwidth windows centered at each RR, downward trends are present in both NIVA and PPG (see Figure 3). There are significant differences $(p<0.05)$ in median between the 6-12 bpm and 12-18 bpm distributions for both PPG and NIVA (see Table 1). We obtain similar results using -3dB wide windows for NIVA. However, the differences are no longer significant for PPG. Using coherence-defined windows, fails at both NIVA and PPG.

\section{Discussion}

The NIVA signal is strongly modulated by breathing. This remains an advantage for estimating RR, a task in which it achieves $100 \%$ accuracy when estimated through PRV, clearly outperforming PPG at the wrist. STT works better for PPG than for NIVA, although not better than PRV. Taking advantage of this, we have used this information to accurately calculate the HF band, of great interest as a marker of the parasympathetic system. In this task, both NIVA and PPG are able to distinguish between groups at different RRs using fixed-width windows. However, only NIVA passes this task when we also leave the choice of window width free. It brings us closer to a real case of sensor application where breathing is unknown. Neither NIVA nor PPG are able to distinguish between groups using the coherence-based method. This may be because STT is not as good estimator of respiration as it is PRV in this particular application. The respiratory peak has been obtained from the PRV instead of from the signal PSD, as in [5], because in the NIVA signal the respiration band is heavily filtered, probably due to the high-pass nature of the derivative, and it is difficult to discriminate a peak there.

Another issue to be addressed is that the breathing signal and tidal volume were not measured during the experiment. Variations in tidal volume are known to affect $P_{H F}$ in the opposite way as changing the RR does. The greater 
Table 1: p-values of the Wilcoxon test. Values below 0.05 are marked with an asterisk (*).

\begin{tabular}{lllllll}
\hline \hline \multirow{2}{*}{ HF window } & \multicolumn{3}{c}{ NIVA $(5 \mathrm{~Hz})$} & \multicolumn{2}{c}{ NIVA $(2 \mathrm{~Hz})$} & \multicolumn{2}{c}{ PPG } \\
& 6 to 12 & 12 to 18 & 6 to 12 & 12 to 18 & 6 to 12 & 12 to 18 \\
\hline Fixed width & $0.008^{*}$ & $0.039^{*}$ & $0.008^{*}$ & $0.016^{*}$ & $0.016^{*}$ & $0.039^{*}$ \\
-3dB width & $0.039^{*}$ & $0.039^{*}$ & $0.008^{*}$ & $0.023^{*}$ & 0.461 & 0.078 \\
Coherence PRV-STT & 0.063 & 0.078 & 0.438 & 0.125 & 0.063 & 0.063 \\
\hline \hline
\end{tabular}

the tidal volume, the greater the $P_{H F}$ and vice versa [8]. Changes in RR are expected to cause changes in tidal volume. This variation should be considered in future experiments. In the absence of a respiratory signal, we have used the metronome frequency as a reference, since in [13] it is shown that the deviations are of the order of tenths of millihertz.

Finally, the database size is a clear limitation. A larger number of subjects should be involved in future research. In this article, we have compared the two signals offered by the device designed by VoluMetrix. However, the combination of both could give additional information that could not be achieved with the signals separately. This is a future line to consider together with comparing performances also in a spontaneous breathing scenario.

\section{Conclusion}

In this work the ability of deriving respiration-guided parasympathetic estimates from a wrist-worn device has been investigated. Respiratory rate was successfully derived in all cases using PRV obtained from NIVA signal, outperforming STT from NIVA and PRV and STT from PPG. $P_{H F}$ from NIVA in a bandwidth dependent on RR estimates, showed a significant decrease with increasing $\mathrm{RR}$, supporting its use as parasympathetic estimate.

\section{Acknowledgments}

This work was supported in part by AEI, FEDER and Gobierno de Aragón, under projects RTI2018-097723-B-I00, LMP44-18 and BSI$\mathrm{CoS}$ group (T39-20R), in part by the European Union's Framework Programme for Research and Innovation Horizon 2020 (2014-2020) under the Marie Skodowska-Curie Grant 745755, and in part by CIBER (Instituto de Salud Carlos III).

\section{Conflict of interest}

Kyle Hocking, PhD, is Founder, CEO and President of VoluMetrix. Colleen Brophy, MD, is Founder and CMO of VoluMetrix. Bret Alvis, $\mathrm{MD}$, owns stock in VoluMetrix. They are inventors on intellectual property in the field of venous waveform analysis assigned to Vanderbilt and licensed to VoluMetrix. Annie Alvis, MBA, owns stock in VoluMetrix and is $\mathrm{COO}$ of VoluMetrix. Bret and Annie Alvis are married.

\section{References}

[1] Gil E, et al. Photoplethysmography pulse rate variability as a surrogate measurement of heart rate variability dur- ing non-stationary conditions. Physiological measurement 2010;31(9):1271.

[2] Peralta E, et al. Optimal fiducial points for pulse rate variability analysis from forehead and finger photoplethysmographic signals. Physiological measurement 2019; 40(2):025007.

[3] Hocking KM, et al. Peripheral venous waveform analysis for detecting hemorrhage and iatrogenic volume overload in a porcine model. Shock 2016;46(4):447-452.

[4] Hocking KM, et al. Peripheral iv analysis (piva) of venous waveforms for volume assessment in patients undergoing haemodialysis. BJA British Journal of Anaesthesia 2017; 119(6):1135-1140.

[5] Hernando D, et al. Validity of venous waveform signal for heart rate variability monitoring. In 2018 Computing in Cardiology Conference (CinC), volume 45. IEEE, 2018; $1-4$.

[6] Hernando D, et al. Effect of yoga on pulse rate variability measured from a venous pressure waveform. In 2019 41st Annual International Conference of the IEEE Engineering in Medicine and Biology Society (EMBC). IEEE, 2019; 372-375.

[7] Hernando A, et al. Inclusion of respiratory frequency information in heart rate variability analysis for stress assessment. IEEE journal of biomedical and health informatics 2016;20(4):1016-1025.

[8] Hirsch JA, Bishop B. Respiratory sinus arrhythmia in humans: how breathing pattern modulates heart rate. American Journal of Physiology Heart and Circulatory Physiology 1981;241(4):H620-H629.

[9] Lázaro J, et al. Deriving respiration from photoplethysmographic pulse width. Medical biological engineering computing 2013;51(1-2):233-242.

[10] Mateo J, Laguna P. Analysis of heart rate variability in the presence of ectopic beats using the heart timing signal. IEEE Transactions on biomedical engineering 2003; 50(3):334-343.

[11] Bailón R, Sornmo L, Laguna P. A robust method for ecgbased estimation of the respiratory frequency during stress testing. IEEE transactions on biomedical engineering 2006; 53(7):1273-1285.

[12] Peláez-Coca MD, et al. Cross time-frequency analysis for combining information of several sources: Application to estimation of spontaneous respiratory rate from photoplethysmography. Computational and mathematical methods in medicine 2013;2013.

[13] Lázaro J, et al. Respiratory rate derived from smartphonecamera-acquired pulse photoplethysmographic signals. Physiological measurement 2015;36(11):2317.

Feel free to contact me at dcajal@unizar.es 